\title{
Electronic Opinion Analysis in Organizational Culture Audit
}

\author{
Shahren Ahmad Zaidi Adruce ${ }^{1,2}$, Abdullah-Al-Jubair ${ }^{1}$, Lee Nung Kion ${ }^{1 *}$ \\ ${ }^{1}$ Faculty of Cognitive Sciences and Human Development, ${ }^{2}$ Institute of Borneo Studies \\ Universiti Malaysia Sarawak, \\ Kota Samarahan, Sarawak, Malaysia
}

\begin{abstract}
Organizational culture defines an organization's uniqueness and identity. It is made up of values, beliefs, attitudes, norms, and patterns of behavior that are shared and adopted by individuals in the organization to cope with internal and external pressure. Computerized culture audit system is more cost efficient, time saving and is less prone to error. However, one of the challenges faced is the difficulty in obtaining accurate employee opinions from free texts. The existing sentiment analysis methods available cannot effectively be applied directly to the organizational culture context for employee opinion analysis. Therefore, this study proposes an employee opinion analysis method known as "Opinion Keyword Extraction" which is based on building customized corpus specific for sentiment analysis in organizational culture context. Opinion Keyword Extraction is a combination of the rule-base and lexicon approach using our own corpus datastore. The customized corpus consists of features related to negation detection, detection of special words relevant to organizational culture and detection of emotion symbols. We evaluated our method using primary data collected from 100 participants and found that our Opinion Keyword Extraction method performed better in comparison with existing methods
\end{abstract}

Keywords: Organizational culture audit, Sentiment analysis, Text mining

\section{INTRODUCTION}

Organization culture represents the collective values, views, expectations, basic assumptions, and means that describe how people really think, agree and execute [2]. An organizational cultural audit (OCA) is an instrument used for understanding organizations, by completing investigations and assessments of an organization's culture [1]. Employee opinion analysis is an important feature of any OCA which helps the management to know more about the current culture and can provide benefits to improve the culture in the future.

The existing manual organizational culture auditing system generally uses survey questionnaires and interviews to perform employee opinion analysis [3]. This method is very time consuming for both data analysis and reporting and is also prone to human errors. Manual auditing is also not cost effective as it requires a lot of paper, printing and physical space for opinion analysis [2].

For a typical medium-sized company it might take months to produce the final audit report. A computerized and automated system is necessary to improve the effectiveness and efficiency of OCA [2].

Sentiment analysis (also known as opinion extraction) refers to the utilization of natural language processing,

*Email Address: nklee@unimas.my text analysis and computational linguistics to find and extract particular information from texts. The output of sentiment analysis is identifying the polarity of texts as positive, negative or neutral towards a certain topic. In the OCA system, texts collected from open ended questions can be valuable feedback to an organization. Sentiment analysis techniques are generally classified into machine learning approach and lexicon based approach. Existing famous sentiment analysis methods include machine learning, lexicon and hybrid approach.

The Machine learning approach (ML) applies supervised or unsupervised learning with collective linguistic features for prediction. The supervised methods utilize a big number of labeled training data. The unsupervised methods are used when finding these labeled training data is difficult. ML approach is usually applied for large datasets such as for reviews of different contexts [15][4]. While effective, ML approach needs a large dataset which is labor demanding to collect.

Lexicon based approach is based on a sentiment lexicon, a group of known and precompiled sentiment terms. It can be classified into dictionary based approach and corpus based approach. The dictionary based approach initially searches for opinion words, and then searches the dictionary for their synonyms and antonyms. The corpus based approach begins with a group of opinion words, and then finds other opinion words in a 